\title{
Time resource management of OAR based on fuzzy logic priority for multiple target tracking
}

\author{
HAN Qinghua ${ }^{1}$, PAN Minghai ${ }^{1}$, ZHANG Wucai ${ }^{1}$, and LIANG Zhiheng ${ }^{2, *}$ \\ 1. College of Electronic and Information Engineering, Nanjing University of Aeronautics and Astronautics, Nanjing 211106, China; \\ 2. Department of Precision Instrument, School of Mechanical Engineering, Tsinghua University, Beijing 100084, China
}

\begin{abstract}
For coping with the multiple target tracking in the presence of complex time-varying environments and unknown target information, a time resource management scheme based on chance-constraint programming (CCP) employing fuzzy logic priority is proposed for opportunistic array radar (OAR). In this scheme, the total beam illuminating time is minimized by effective time resource allocation so that the desired tracking performance is achieved. Meanwhile, owing to the randomness of radar cross section (RCS), the CCP is used to balance tracking accuracy and time resource conditioned on the specified confidence level. The adaptive fuzzy logic prioritization, imitating the human decision-making process for ranking radar targets, can realize the full potential of radar. The Bayesian Cramér-Rao lower bound (BCRLB) provides us with a low bound of localization estimation root-mean-square error (RMSE), and equally important, it can be calculated predictively. Consequently, it is employed as an optimization criterion for the time resource allocation scheme. The stochastic simulation is integrated into the genetic algorithm (GA) to compose a hybrid intelligent optimization algorithm to solve the CCP optimization problem. The simulation results show that the time resource is saved strikingly and the radar performance is also improved.
\end{abstract}

Keywords: chance-constraint programming (CCP), fuzzy logic priority, opportunistic array radar (OAR), root-mean-square error (RMSE), Bayesian Cramér-Rao lower bound (BCRLB).

DOI: $10.21629 /$ JSEE.2018.04.09

\section{Introduction}

Opportunistic array radar (OAR) proposed by the United States Naval Postgraduate School (NPS) is a new system radar for next generation naval stealth destroyer $\mathrm{DD}(\mathrm{X})$ $[1-3]$. In OAR, the stealth of the military platform is taken as the core and the digital array is regarded as the base, and then the array elements and the transmit/receive (T/R) modules are placed arbitrarily and aperiodically at available open areas over the entire 3-D space of the platform

\footnotetext{
Manuscript received March 16, 2017.

*Corresponding author.

This work was supported by the National Natural Science Foundation of China (61271327; 61671241).
}

$[4,5]$. Due to the unique structure of the antenna array of OAR, we can realize that parts of the array elements are in activation state while the others are shut down. Hence, multiple orthogonal beams can be generated simultaneously with various desired beam patterns [6,7]. However, in the most stressing conditions, the targets are so many that there are insufficient beams to track all the targets, and the competition for limited beam resources onboard the military platform remains unabated. Therefore, the conflicts between targets have to be resolved when the radar system operates in an environment that leads to an overload situation.

The time resource management is a rational allocation of dwell time over all the targets, or can be called beam dwell scheduling policy $[8,9]$. Generally speaking, two kinds of time resource management policies are considered. In the first policy, an optimal time resource allocation is selected so that the achievable localization rootmean-square error (RMSE) is minimized; in the second policy, the total illuminating time used for the estimation process is minimized for the desired tracking accuracy threshold [10]. However, when the radar systems are installed on the mobile platforms operating over prolonged time periods with limited resources, we merely need to obtain a desired performance level instead of minimizing the tracking error as much as possible [11], i.e., the latter type might be more useful in practical work.

In the traditional study of radar resource management, the radar cross section (RCS) is taken as a determined value at every instant while the targets are illuminated $[11,12]$. In practical target tracking process, attributing to the significantly variable environment and the ambiguous target information, the target RCS is uncertain [13], i.e., the RCS is a random variable. Therefore, we introduce chance-constraint programming (CCP) [14] to achieve a trade-off between time resource and tracking accuracy. Through this CCP, the total tracking accuracy could be attained under a certain confidence level so that the time resource for tracking would be reduced more.

When the aforementioned time resource CCP model is 
built, the priority of the targets is different. In the previous study, the fixed prioritization method for ranking targets is applied in the proposed resource management policies $[15,16]$, or the nonrepresentational concepts [17], such as importance, urgency and validity, are attached to the radar targets instead of considering the concrete concepts, for instance, velocity, range, azimuth and height, etc. These methodologies cannot intelligently determine the priority of radar targets by a clear concept. Nevertheless, the fuzzy logic adaptive prioritization approach is an intelligent and effective method to calculate the priority of radar targets [18-20]. Considering the dynamic characteristics of target tracking, the fuzzy logic adaptive prioritization approach can imitate the human decision-making process and provide an intelligent and efficient algorithm to rank the targets.

Besides the above preparation, we also need to find a variable as the measurement criteria of the tracking accuracy of radar targets for pre-allocating time resources. And this problem has been addressed in published references of resource management [21-26]. The recursive Bayesian Cramér-Rao lower bound (BCRLB) was first derived in [21], where an elegant method is provided for computing Bayesian information matrix (BIM) avoiding manipulating large matrices. An upgraded derivation which is applicable to the nonlinear dynamics process is contained in [22], and the contributions of the prior information matrix and the data information matrix are separated by this derivation. The BCRLB is wildly applied to the resource management of radar system by the simplified computing method, and excellent results are obtained [23-26].

On the basis of the aforementioned study, in this paper, we propose a time resource management scheme based on $\mathrm{CCP}$ with multibeam working mode employing fuzzy logic priority for tracking multiple, broadly distributed and independent point targets. It is assumed that only one target is in the region illuminated by a beam at every instant, however, a beam can successively track multiple targets in every frame. Though the aim is to minimize the total illuminating time of all the beams conditioned on the specified tracking performance threshold, the total illuminating time of one beam can be minimized by rational combinatorial optimization of radar targets among the beams. Meanwhile, the fuzzy if-then inference rules are invited to calculate the fuzzy logic priority of radar targets, and the number of fuzzy rules required to assess the value of influence factors plays an important role in guaranteeing the veracity and smoothness of the prioritization curves. The whole algorithm can be viewed as an intelligent response of radar systems to multiple target tracking by perceiving the environments. The BCRLB, measuring the estimated RMSE of target tracking accuracy, provides us a criterion for pre-allocating the time resource. By integrating stochastic simulation and the genetic algorithm (GA), the optimal solutions can be derived.

This paper is organized as follows. The system model is introduced in Section 2. In Section 3, the random CCP model combining fuzzy logic inference system is formulated. Section 4 proposes an approach for solving the model. Several numerical results are provided in Section 5. Finally, the conclusions and future work of this paper are given in Section 6.

\section{System model}

We consider 2-dimensional multiple target tracking employing a colocated OAR, located at $\left(x_{0}, y_{0}\right)$. This system adopts a multibeam concept in which multiple simultaneous and independent beams are synthesized by different probing signals from various colocated transmitters. Hence, the OAR can generate $M$ orthogonal transmit beams simultaneously. One beam can only cover one target at every instant. Let $T_{0}$ denote the time interval of successive frames, which is regarded as the tracking sample interval. Then the $q$ th $(q=1,2, \ldots, Q$ and $Q \geqslant M)$ target at time $k T_{0}$ is located at $\left(x_{k}^{q}, y_{k}^{q}\right)(k=0,1,2, \ldots, K)$, and moves at the speed of $\left(\dot{x}_{k}^{q}, \dot{y}_{k}^{q}\right)$. The multiple target tracking sketch is shown in Fig. 1.

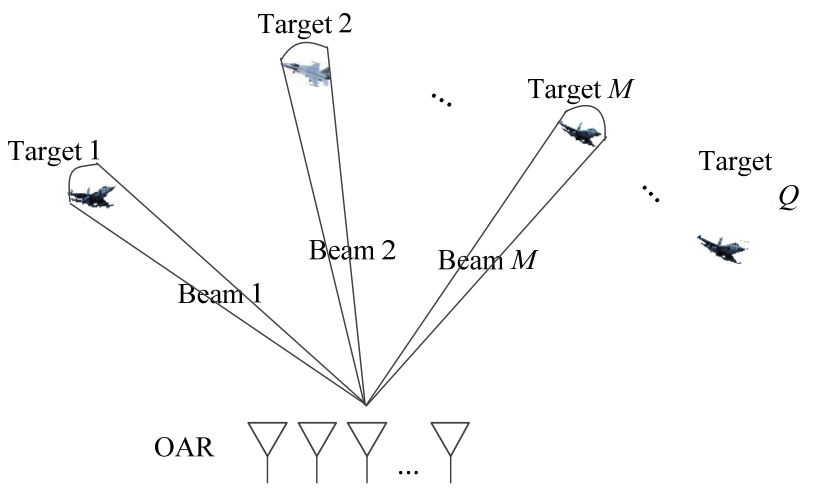

Fig. 1 Multiple target tracking sketch of OAR

\subsection{Signal model}

It is assumed that the transmitting signal of OAR to the $q$ th target at the $k$ th sample interval is

$$
s_{k}^{q}(t)=\sqrt{p_{k}^{q}} S_{k}^{q}(t) \exp \left(-\mathrm{j} 2 \pi f_{\mathrm{c}} t\right)
$$

where $p_{k}^{q}$ denotes the transmitting power to the $q$ th target at the $k$ th sample interval, and $f_{c}$ is the carrier frequency. $S_{k}^{q}(t)$ denotes the complex envelope of the transmitting signal, and its effective bandwidth [27] is

$$
\left(B_{k}^{q}\right)^{2}=\frac{\int_{-\infty}^{+\infty}(f-\bar{f})^{2}\left|S_{k}^{q}(f)\right|^{2} \mathrm{~d} f}{\int_{-\infty}^{+\infty}\left|S_{k}^{q}(f)\right|^{2} \mathrm{~d} f}
$$


where $\bar{f}$ represents the first order origin moment of frequency spectrum $\left|S_{k}^{q}(f)\right|^{2}$, and in general, $\bar{f}=0$. The effective time duration of $S_{k}^{q}(t)$ [27] is

$$
\left(T_{k}^{q}\right)^{2}=\frac{\int_{-\infty}^{+\infty}(t-\bar{t})^{2}\left|S_{k}^{q}(t)\right|^{2} \mathrm{~d} t}{\int_{-\infty}^{+\infty}\left|S_{k}^{q}(t)\right|^{2} \mathrm{~d} t}
$$

where $\bar{t}$ represents the first order origin moment of $\left|S_{k}^{q}(t)\right|^{2}$, and in general, $\bar{t}=0$. The effective time duration $T_{k}^{q}$ of each target is impacted by the illuminating time of the corresponding beam, which is allocated rationally and optimally over all the targets.

The baseband representation for the echo signal from the $q$ th target at the $k$ th sample interval is

$$
\begin{gathered}
r_{k}^{q}(t)=h_{k}^{q} \sqrt{\alpha_{k}^{q} p_{k}^{q}} S_{k}^{q}\left(t-\tau_{k}^{q}\right) . \\
\exp \left(-\mathrm{j} 2 \pi f_{k}^{q} t\right)+w_{k}^{q}(t)
\end{gathered}
$$

where $h_{k}^{q}$ denotes the target RCS, which is a random variable [13]. $\alpha_{k}^{q} \propto 1 /\left(R_{k}^{q}\right)^{4}$ denotes the variation in the signal strength due to the path loss effect along the path OARtarget $q$-OAR. The time delay is $\tau_{k}^{q}$ and the Doppler frequency is $f_{k}^{q}$. $w_{k}^{q}(t)$ is a zero-mean, complex Gaussian white noise.

\subsection{Motion model}

The $q$ th target moves rectilinearly with a constant velocity in the $x y$ plane:

$$
\boldsymbol{\xi}_{k}^{q}=\boldsymbol{F}_{q} \boldsymbol{\xi}_{k-1}^{q}+\boldsymbol{v}_{k-1}^{q}
$$

where $\boldsymbol{\xi}_{k}^{q}=\left[\begin{array}{llll}x_{k}^{q} & \dot{x}_{k}^{q} & y_{k}^{q} & \dot{y}_{k}^{q}\end{array}\right]^{\mathrm{T}}$ denotes the state vector of the $q$ th target at the $k$ th sample interval. $\boldsymbol{F}_{q}$ is the $4 \times 4$ transition matrix:

$$
\boldsymbol{F}_{q}=\left[\begin{array}{cccc}
1 & T_{0} & 0 & 0 \\
0 & 1 & 0 & 0 \\
0 & 0 & 1 & T_{0} \\
0 & 0 & 0 & 1
\end{array}\right] .
$$

$\boldsymbol{v}_{k-1}^{q}$ represents a zero-mean, complex Gaussian white noise, and its covariance is

$$
\boldsymbol{Q}_{k-1}^{q}=I_{q}\left[\begin{array}{cccc}
\frac{1}{3} T_{0}^{3} & \frac{1}{2} T_{0}^{2} & 0 & 0 \\
\frac{1}{2} T_{0}^{2} & T_{0} & 0 & 0 \\
0 & 0 & \frac{1}{3} T_{0}^{3} & \frac{1}{2} T_{0}^{2} \\
0 & 0 & \frac{1}{2} T_{0}^{2} & T_{0}
\end{array}\right]
$$

where $I_{q}$ is the process noise intensity of the $q$ th target [27].

\subsection{Measurement model}

The received signal from the $q$ th target is an attenuated version of the transmitted signal. The range, azimuth and Doppler frequency can be extracted from the received signal. The nonlinear measurement equation can be written as

$$
\boldsymbol{z}_{k}^{q}=\boldsymbol{g}\left(\boldsymbol{\xi}_{k}^{q}\right)+\boldsymbol{w}_{k}^{q}
$$

where

$$
\boldsymbol{g}\left(\boldsymbol{\xi}_{k}^{q}\right)=\left(R_{k}^{q}, \theta_{k}^{q}, f_{k}^{q}\right)^{\mathrm{T}} .
$$

$R_{k}^{q}$ denotes the range between the OAR and the $q$ th target, and it can be calculated by the time delay $\tau_{k}^{q}$.

$$
R_{k}^{q}=\frac{c \tau_{k}^{q}}{2}=\sqrt{\left(x_{k}^{q}-x_{0}\right)^{2}+\left(y_{k}^{q}-y_{0}\right)^{2}}
$$

where $c$ is the light speed. The azimuth $\theta_{k}^{q}$ can be expressed as

$$
\theta_{k}^{q}=\arctan \left[\left(y_{k}^{q}-y_{0}\right) /\left(x_{k}^{q}-x_{0}\right)\right] .
$$

$f_{k}^{q}$ is the Doppler shift determined by the target coordinates and the velocity:

$$
f_{k}^{q}=-\frac{2}{\lambda_{k}^{q}}\left(\dot{x}_{k}^{q}, \dot{y}_{k}^{q}\right) \cdot\left(x_{k}^{q}, y_{k}^{q}\right)^{\mathrm{T}} / R_{k}^{q}
$$

where $(\cdot)^{\mathrm{T}}$ denotes matrix transposition, and $\lambda_{k}^{q}$ denotes the wavelength.

$\boldsymbol{w}_{k}^{q}$ is a zero-mean and Gaussian white noise, and its covariance is

$$
\boldsymbol{R}_{\boldsymbol{\xi}_{k}^{q}}=\operatorname{blkdiag}\left(\sigma_{R_{k}^{q}}^{2} \quad \sigma_{\theta_{k}^{q}}^{2} \quad \sigma_{f_{k}^{q}}^{2}\right)
$$

where $\operatorname{blkdiag}(\cdot)$ denotes block diagonal matrix. $\sigma_{R_{k}^{q}}^{2}, \sigma_{\theta_{k}^{q}}^{2}$ and $\sigma_{f_{k}^{q}}^{2}$ are the BCRLB of MSE of the range, azimuth and Doppler shift at high signal-to-noise ratio (SNR). The BCRLB for $\sigma_{R_{k}^{q}}^{2}$ and $\sigma_{f_{k}^{q}}^{2}$ are given in Chapter 10 of [27] and the BCRLB for $\sigma_{\theta_{k}^{q}}^{2}$ follows from [28]. The number of coherent pulses illuminating the $q$ th target is $L_{k}^{q}$, and $T_{1}$ is the effective time duration of a signal coherent pulse. Then the BCRLB of MSE of the range, azimuth and Doppler shift can be described as follows:

$$
\left\{\begin{array}{l}
\sigma_{R_{k}^{q}}^{2} \propto\left(L_{k}^{q}(S / N)_{k}^{q} \cdot\left(B_{k}^{q}\right)^{2}\right)^{-1} \\
\sigma_{\theta_{k}^{q}}^{2} \propto\left(L_{k}^{q}(S / N)_{k}^{q} /\left(B_{N N}\right)^{2}\right)^{-1} \\
\sigma_{f_{k}^{q}}^{2} \propto\left(L_{k}^{q}(S / N)_{k}^{q} \cdot\left(L_{k}^{q} \cdot T_{1}\right)^{2}\right)^{-1}
\end{array}\right.
$$

where $B_{N N}$ is the null-to-null beamwidth of the receiver antenna. $(S / N)_{k}^{q}$ is the SNR of the echo signal of a single coherent pulse from the $q$ th target at the $k$ th sample interval:

$$
(S / N)_{k}^{q} \propto \alpha_{k}^{q}\left|h_{k}^{q}\right|^{2} p_{k}^{q} .
$$

Hence, the measurement equation can be transformed as follows:

$$
\boldsymbol{z}_{k}^{q}=\left(\widetilde{R}_{k}^{q}, \widetilde{\theta}_{k}^{q}, \widetilde{f}_{k}^{q}\right)^{\mathrm{T}}=
$$




$$
\left(R_{k}^{q}, \theta_{k}^{q}, f_{k}^{q}\right)^{\mathrm{T}}+\left(\Delta R_{k}^{q}, \Delta \theta_{k}^{q}, \Delta f_{k}^{q}\right)^{\mathrm{T}}
$$

where $\Delta R_{k}^{q}, \Delta \theta_{k}^{q}$ and $\Delta f_{k}^{q}$ are the error information of the range, azimuth and Doppler shift, respectively.

\subsection{Fuzzy logic adaptive prioritization}

By other sensors, other operating modes or a tracking algorithm of OAR, we can obtain the information for assigning priority of tracked targets. On the basis of the information, the fuzzy logic adaptive prioritization method can calculate the priority of tracked targets intelligently as the human decision-making process.

\subsubsection{Fuzzy values of fuzzy variables}

The decision tree $[19,20]$ is used for evaluating the priority of tracked targets. The influence factors of the priority contain the range, velocity, height, azimuth, range rate, identity, manoeuvre and weapon system, etc. In order to simplify the calculation, three different fuzzy variables providing information concerning the priority are considered: range, velocity and threat. 'Threat' is the fuzzy variable which represents the degree of threat of a target according to its trajectory and identity. The exactness of priority is proportional to the number of fuzzy values of each fuzzy variable. We attribute seven fuzzy values, three fuzzy values, three fuzzy values and seven fuzzy values to the fuzzy variables 'range', 'velocity', 'threat' and 'priority', respectively. The detailed definition of fuzzy values are listed in Table $1[19,20]$.

Table 1 Fuzzy values related to fuzzy variables

\begin{tabular}{cc}
\hline Fuzzy variable & Fuzzy value \\
\hline Range & $\begin{array}{c}\text { Very close, close, medium-close, medium, } \\
\text { medium-far, far, very far }\end{array}$ \\
\hline Velocity & Slow, medium, fast \\
\hline Threat & Low, medium, high \\
\hline Priority & $\begin{array}{c}\text { Very low, low, medium-low, medium, } \\
\text { medium-high, high, very high }\end{array}$ \\
\hline
\end{tabular}

The four fuzzy variables change within their respective domains: 'range' changes within $0-150 \mathrm{~km}$; 'velocity' changes within $0-900 \mathrm{~m} / \mathrm{s}$; 'threat' and 'priority' both change within $0-1$. The triangular fuzzy number is used to represent the membership function of fuzzy values of fuzzy variables. The membership functions, which are employed for the fuzzy values of 'range', 'velocity', 'threat' and 'priority', are presented in Fig. 2.

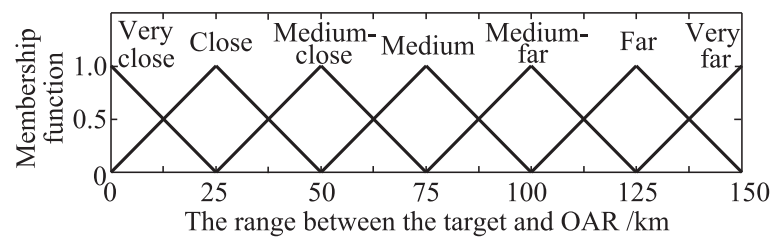

(a) Range

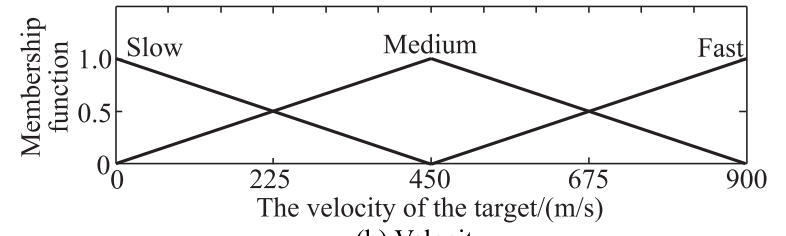

(b) Velocity

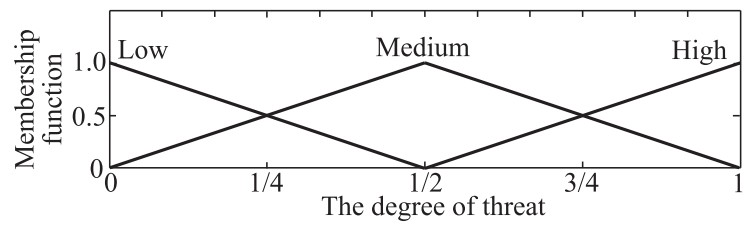

(c) Threat

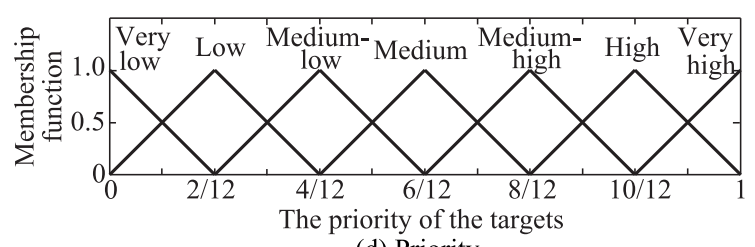

(d) Priority

Fig. 2 Membership function related to fuzzy variables

\subsubsection{Fuzzy rules base}

In Table 1, based on the permutation and combination, it can be seen that there are 63 combinations of fuzzy values. Hence, on the basis of intuitive and expert considerations and then tuned by simulation tests, 63 if-then inferential rules are used here.

\subsubsection{Fuzzy logic inference system}

In order to meet the needs of practical work, the input and output of fuzzy logic inference system are both exact values. However, the operational objectives of fuzzy inference are fuzzy values. As a consequence, in order to transform the exact values into fuzzy values, we need to fuzzify the input values. Meanwhile, on the contrary, the output values of fuzzy inference need to be defuzzified for allocating the time resource during the target tracking process, which needs the exact values of 'priority'. Hence, an integrated fuzzy logic inference system to calculate the priority of the targets is built in Fig. 3 [29].

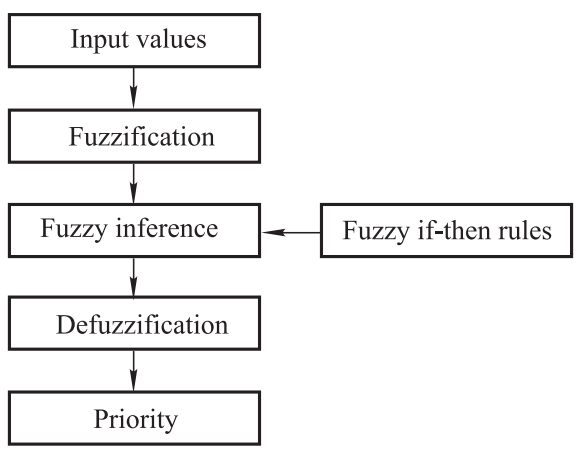

Fig. 3 Integrated fuzzy logic inference system 
Through this system, the priority of targets can be generated jointly by the range, velocity and threat of targets.

\section{Chance-constraint programming model}

\subsection{BCRLB}

It is assumed that $\widehat{\boldsymbol{\xi}}_{k}^{q}$ is an estimation of state vector $\boldsymbol{\xi}_{k}^{q}$. Hence, $\widehat{\boldsymbol{\xi}}_{k}^{q}$ is a function of the measurement value $\boldsymbol{z}_{k}^{q}$. In the Bayesian estimation problem, the BCRLB $C_{\mathrm{BCRLB}}$ provides a low bound for the MSE in estimating the random vector $\boldsymbol{\xi}_{k}^{q}$, and it is the inverse of BIM $\boldsymbol{J}\left(\boldsymbol{\xi}_{k}^{q}\right)$. The MSE of any estimator cannot be less than the BCRLB $C_{\mathrm{BCRLB}}$ [30]:

$$
\begin{gathered}
\mathcal{E}\left[\left(\widehat{\boldsymbol{\xi}}_{k}^{q}\left(\boldsymbol{z}_{k}^{q}\right)-\boldsymbol{\xi}_{k}^{q}\right)\left(\widehat{\boldsymbol{\xi}}_{k}^{q}\left(\boldsymbol{z}_{k}^{q}\right)-\boldsymbol{\xi}_{k}^{q}\right)^{\mathrm{T}}\right] \geqslant \\
\boldsymbol{C}_{\mathrm{BCRLB}}\left(\boldsymbol{\xi}_{k}^{q}\right)=\boldsymbol{J}^{-1}\left(\boldsymbol{\xi}_{k}^{q}\right)
\end{gathered}
$$

where $\mathcal{E}[\cdot]$ denotes expectation with respect to the joint probability density function (PDF) $p\left(\boldsymbol{z}_{k}^{q}, \boldsymbol{\xi}_{k}^{q}\right)$ of $\boldsymbol{z}_{k}^{q}$ and $\boldsymbol{\xi}_{k}^{q}$. The matrix inequality means that

$$
\mathcal{E}\left[\left(\widehat{\boldsymbol{\xi}}_{k}^{q}\left(\boldsymbol{z}_{k}^{q}\right)-\boldsymbol{\xi}_{k}^{q}\right)\left(\widehat{\boldsymbol{\xi}}_{k}^{q}\left(\boldsymbol{z}_{k}^{q}\right)-\boldsymbol{\xi}_{k}^{q}\right)^{\mathrm{T}}\right]-\boldsymbol{C}_{\mathrm{BCRLB}}\left(\boldsymbol{\xi}_{k}^{q}\right)
$$

is a nonnegative definite matrix. The BIM $\boldsymbol{J}\left(\boldsymbol{\xi}_{k}^{q}\right)$ can be described as

$$
\begin{gathered}
\boldsymbol{J}\left(\boldsymbol{\xi}_{k}^{q}\right)= \\
\mathcal{E}\left(\left(\frac{\partial \ln p\left(\boldsymbol{z}_{k}^{q}, \boldsymbol{\xi}_{k}^{q}\right)}{\partial \boldsymbol{\xi}_{k}^{q}}\right)\left(\frac{\partial \ln p\left(\boldsymbol{z}_{k}^{q}, \boldsymbol{\xi}_{k}^{q}\right)}{\partial \boldsymbol{\xi}_{k}^{q}}\right)^{\mathrm{T}}\right) .
\end{gathered}
$$

The joint PDF $p\left(\boldsymbol{z}_{k}^{q}, \boldsymbol{\xi}_{k}^{q}\right)$ can be replaced by the product of $\operatorname{PDF} p\left(\boldsymbol{\xi}_{k}^{q}\right)$ and conditional PDF $p\left(\boldsymbol{z}_{k}^{q} \mid \boldsymbol{\xi}_{k}^{q}\right)$.

$$
p\left(\boldsymbol{z}_{k}^{q}, \boldsymbol{\xi}_{k}^{q}\right)=p\left(\boldsymbol{\xi}_{k}^{q}\right) p\left(\boldsymbol{z}_{k}^{q} \mid \boldsymbol{\xi}_{k}^{q}\right)
$$

Hence, the BIM $\boldsymbol{J}\left(\boldsymbol{\xi}_{k}^{q}\right)$ can be divided into a prior information matrix $\boldsymbol{J}_{\mathrm{P}}\left(\boldsymbol{\xi}_{k}^{q}\right)$ and a data information matrix $\boldsymbol{J}_{\mathrm{D}}\left(\boldsymbol{\xi}_{k}^{q}\right)[30]$.

$$
\boldsymbol{J}\left(\boldsymbol{\xi}_{k}^{q}\right)=\boldsymbol{J}_{\mathrm{P}}\left(\boldsymbol{\xi}_{k}^{q}\right)+\boldsymbol{J}_{\mathrm{D}}\left(\boldsymbol{\xi}_{k}^{q}\right)
$$

The prior information $\boldsymbol{J}_{\mathrm{P}}\left(\boldsymbol{\xi}_{k}^{q}\right)$ is determined by the motion equations of targets, and the beam illuminating time has no impact on it. On the contrary, the data information $\boldsymbol{J}_{\mathrm{D}}\left(\boldsymbol{\xi}_{k}^{q}\right)$ is affected by the total illuminating time allocated to the targets. And the longer the radar illuminates the target, the larger the $\boldsymbol{J}_{\mathrm{D}}\left(\boldsymbol{\xi}_{k}^{q}\right)$ is.

According to [30,31], the complete BIM $\boldsymbol{J}\left(\boldsymbol{\xi}_{k}^{q}\right)$ can be obtained.

$$
\begin{gathered}
\boldsymbol{J}\left(\boldsymbol{\xi}_{k}^{q}\right)=\boldsymbol{J}_{\mathrm{P}}\left(\boldsymbol{\xi}_{k}^{q}\right)+\boldsymbol{J}_{\mathrm{D}}\left(\boldsymbol{\xi}_{k}^{q}\right)= \\
{\left[\boldsymbol{Q}_{k-1}^{q}+\boldsymbol{F}_{q} \boldsymbol{J}^{-1}\left(\boldsymbol{\xi}_{k-1}^{q}\right) \boldsymbol{F}_{q}^{\mathrm{T}}\right]^{-1}+\left.\boldsymbol{G}_{\boldsymbol{\xi}_{k}^{q}}^{\mathrm{T}} \boldsymbol{R}_{\boldsymbol{\xi}_{k}^{q}}^{-1} \boldsymbol{G}_{\boldsymbol{\xi}_{k}^{q}}\right|_{\boldsymbol{\xi}_{k \mid k-1}^{q}}}
\end{gathered}
$$

where $\boldsymbol{G}_{\boldsymbol{\xi}_{k}^{q}}$ and $\boldsymbol{R}_{\boldsymbol{\xi}_{k}^{q}}$ denote the Jacobian matrix and the measurement covariance matrix evaluated at $\boldsymbol{\xi}_{k \mid k-1}^{q}$, respectively. $\boldsymbol{\xi}_{k \mid k-1}^{q}$ denotes the predicted state vector of the $q$ th target for the case of zero process noise [32].

\subsection{Establishment of CCP model based on fuzzy logic adaptive prioritization}

Through the derivation of Section 3.1, the BCRLB $\boldsymbol{C}_{\mathrm{BCRLB}}\left(\boldsymbol{\xi}_{k}^{q}\right)$ can be got as the inverse matrix of BIM $\boldsymbol{J}\left(\boldsymbol{\xi}_{k}^{q}\right)$. The diagonal elements of the BCRLB $\boldsymbol{C}_{\mathrm{BCRLB}}\left(\boldsymbol{\xi}_{k}^{q}\right)$ denote the low bound of MSE of the state vector estimation. As shown in (21), the BCRLB $\boldsymbol{C}_{\mathrm{BCRLB}}\left(\boldsymbol{\xi}_{k}^{q}\right)$ of the $q$ th target can be predicted by recursive computation. Firstly, we pre-allocate the time resource over all the targets. Then, in conjunction with the predicted target positions, the data information matrix $\boldsymbol{J}_{\mathrm{D}}\left(\boldsymbol{\xi}_{k}^{q}\right)$ can be deduced. Together with the BIM $\boldsymbol{J}\left(\boldsymbol{\xi}_{k-1}^{q}\right)$ of time $k-1$, the BIM $\boldsymbol{J}\left(\boldsymbol{\xi}_{k}^{q}\right)$ would be calculated, thus the BCRLB $\boldsymbol{C}_{\mathrm{BCRLB}}\left(\boldsymbol{\xi}_{k}^{q}\right)$ would be also obtained. Based on the aforementioned, in order to build a resource allocation model conveniently and represent the tracking performance of each target accurately, the tracking BCRLB of the $q$ th target can be represented through the square root of the sum of the elements on the leading diagonal:

$$
\begin{gathered}
F\left(T_{k}^{q}, \boldsymbol{\xi}_{k}^{q}, h_{k}^{q}\right)= \\
\sqrt{\operatorname{trace}\left(\boldsymbol{C}_{\mathrm{BCRLB}}\left(T_{k}^{q}, \boldsymbol{\xi}_{k}^{q}, h_{k}^{q}\right)\right)}
\end{gathered}
$$

where $T_{k}^{q}$ denotes the dwell time of the $q$ th target at the $k$ th sample interval. $h_{k}^{q}$ denotes the RCS of the $q$ th target at the $k$ th sample interval.

Herein, the tracking BCRLB of a single target is got. Suppose the priority of all the targets is the same. Then we can sum the tracking BCRLB of all the targets together for the total tracking BCRLB. In accordance with (21), the tracking BCRLB of the targets is related to many parameters. The dwell time vector $\boldsymbol{T}_{k}$ is concerned in this paper. In the actual tracking process, the total illuminating time, in which the beams illuminate the targets, is limited. In certain overload situations, to assure that the tasks can be accomplished as many as possible in the limited tracking time, the resource management model is built to minimize the total illuminating time conditioned on the specified total tracking error threshold. Therefore the conventional resource management model is got.

$$
\begin{gathered}
\min \sum_{q=1}^{Q} T_{k}^{q} \\
\text { s.t. } T_{k}^{q} \geqslant T_{\min } \\
\sum_{q=1}^{Q} F\left(T_{k}^{q}, \boldsymbol{\xi}_{k}^{q}, h_{k}^{q}\right) \leqslant \eta_{0}
\end{gathered}
$$


where $T_{\min }$ is the minimum of the dwell time of each target. $\eta_{0}$ is the specified total tracking error threshold.

Due to the difference of targets, the priority of targets is different. The priority can be reflected by the tracking BCRLB with different weight coefficients. Hence, in view of the priority of the targets, the resource management model can be reformulated as

$$
\begin{gathered}
\min \sum_{q=1}^{Q} T_{k}^{q} \\
\text { s.t. } T_{k}^{q} \geqslant T_{\min } \\
\sum_{q=1}^{Q}\left[w_{k}^{q} \cdot F\left(T_{k}^{q}, \boldsymbol{\xi}_{k}^{q}, h_{k}^{q}\right)\right] \leqslant \eta_{0}
\end{gathered}
$$

where $w_{k}^{q}$ is the weight coefficient of the $q$ th target at the $k$ th sample interval.

In practice, the target RCS is related to identification, attitude and position of the target, and also it is impacted by aspect angle, polarization and so on [13], i.e., the target RCS is unknown and uncertain. Consequently, the target RCS is regarded as a random variable in this paper. Then the deterministic resource allocation model cannot show the characteristic of the targets well and truly, and it cannot guarantee the robustness of the model either. In view of the above situation, the random CCP of the time resource management is introduced [33]. According to (24), the resource management model can be transformed into

$$
\begin{gathered}
\min \sum_{q=1}^{Q} T_{k}^{q} \\
\text { s.t. } T_{k}^{q} \geqslant T_{\min } \\
\operatorname{Pr}\left\{\sum_{q=1}^{Q}\left[w_{k}^{q} \cdot F\left(T_{k}^{q}, \boldsymbol{\xi}_{k}^{q}, h_{k}^{q}\right)\right] \leqslant \eta_{0}\right\} \geqslant \alpha .
\end{gathered}
$$

Because the coherent pulse is used in this paper, the dwell time $T_{k}^{q}$ can be factorized as

$$
T_{k}^{q}=L_{k}^{q} \cdot T_{1}
$$

The model (25) is equivalently transformed to

$$
\begin{gathered}
\min \sum_{q=1}^{Q} L_{k}^{q} \\
\text { s.t. } L_{k}^{q} \geqslant L_{\min } \\
\operatorname{Pr}\left\{\sum_{q=1}^{Q}\left[w_{k}^{q} \cdot F\left(L_{k}^{q}, \boldsymbol{\xi}_{k}^{q}, h_{k}^{q}\right)\right] \leqslant \eta_{0}\right\} \geqslant \alpha .
\end{gathered}
$$

The confidence level $\alpha$ in (25) and (27) is a probability at which it is desired that the stochastic constraint holds. Not only the identity, motion state, and number of the targets, but also the target environments and the amount of radar resources are the factors which impact the confidence level. Thus the selection of the confidence level is a result of synthesizing all kinds of factors.

As described at the beginning of Section 2, the number of targets is more than the number of beams. Hence, the targets should be allocated among the beams appropriately to ensure that the difference of illuminating time between all the beams is as small as possible. We can allocate the $Q$ targets among the $M$ beams like the following description.

Firstly, the dwell time of the $Q$ targets is sorted from large to small. Then the top $M$ elements of the sorted sequence are selected corresponding to the $M$ beams. Thirdly, only the maximum element is selected from the remaining sequence each time to be added to the minimum time of the beams until the remaining elements are all allocated.

\section{Theory and method for solving the model}

In this section, we firstly describe the solving process of the fuzzy logic inference system detailedly. And then the hybrid intelligent optimization algorithm for solving the time resource CCP model is presented. Its object is to predict the optimal time resource allocation for next sample instant. Finally, we integrate the unscented Kalman filter (UKF) and the hybrid intelligent optimization algorithm to produce a target state estimation algorithm for the multiple target tracking.

\subsection{Stochastic simulation}

In order to verify whether the decision vector satisfies the confidence level, the stochastic simulation [33] for (27) is described as follows:

Step 1 Set $N^{\prime}=0$.

Step 2 Generate RCS vector $\left(\boldsymbol{h}_{k}\right)_{i}(i=1,2, \ldots, N)$ of all the targets from their own probability space $(\Omega, \mathcal{A}, \operatorname{Pr})_{q}$.

Step 3 If $\sum_{q=1}^{Q}\left[F\left(L_{k}^{q}, \boldsymbol{\xi}_{k}^{q}, h_{k}^{q}\right)\right] \leqslant \eta_{0}$, then $N^{\prime} \leftarrow N^{\prime}+1$.

Step 4 Repeat the second and third steps $N$ times.

Step $5 \operatorname{Pr}\left(\boldsymbol{L}_{k}\right)=N^{\prime} / N$.

Step 6 If $\operatorname{Pr}\left(\boldsymbol{L}_{k}\right) \geqslant \alpha$, the decision vector $\boldsymbol{L}_{k}$ satisfies the constraint condition; otherwise it does not.

\subsection{Fuzzy logic inference system for calculating the priority}

In the light of Fig. 3, the fuzzy logic inference system consists of four function modules: fuzzification, fuzzy infer- 
ence, fuzzy if-then rules, and defuzzification. The solving method of each module would be elaborated [29,34,35].

\subsubsection{Fuzzification}

When the exact values are input, they should be fuzzified into the fuzzy values. In consequence, the essence of fuzzification is to transform the exact values into fuzzy values. The triangular fuzzifier is used to fuzzify the input values [35].

The triangular fuzzifier maps a real-value point $x^{*} \in U$ into a fuzzy set $A^{\prime}$ in $U$, which has the following triangular membership function:

$$
\mu_{A^{\prime}}(x)=\left\{\begin{array}{l}
1-\frac{\left|x-x^{*}\right|}{\sigma}, \quad\left|x-x^{*}\right| \leqslant \sigma . \\
0, \text { otherwise }
\end{array}\right.
$$

\subsubsection{Fuzzy inference}

Through the triangular fuzzifier, the exact values are changed to fuzzy values. The fuzzy if-then inference rules are given in Section 2.4.2. Then we can use multiconditional fuzzy inference to compute the fuzzy value of the priority.

Suppose the number of fuzzy rules is $N$. Then the general schema of multi-conditional fuzzy inference is shown in Table 2.

\begin{tabular}{cc} 
Table 2 General schema of multi-conditional fuzzy inference \\
\hline Rule number & Fuzzy rules \\
\hline Rule 1 & If $\left(A_{1}\right.$ AND $B_{1}$ AND $\left.C_{1}\right)$, then $D_{1} ;$ \\
Rule 2 & If $\left(A_{2}\right.$ AND $B_{2}$ AND $\left.C_{2}\right)$, then $D_{2} ;$ \\
Rule 3 & If $\left(A_{3}\right.$ AND $B_{3}$ AND $\left.C_{3}\right)$, then $D_{3} ;$ \\
$\vdots$ & $\vdots$ \\
Rule $n$ & If $\left(A_{N}\right.$ AND $B_{N}$ AND $\left.C_{N}\right)$, then $D_{N} ;$ \\
Fact & If $\left(A^{\prime}\right.$ AND $B^{\prime}$ AND $\left.C^{\prime}\right)$ \\
\hline Conclusion & then $D^{\prime}$ \\
\hline
\end{tabular}

In Table 2, $A_{i}$ and $A^{\prime}$ are the fuzzy subsets of the universe $X, B_{i}$ and $B^{\prime}$ are the fuzzy subsets of the universe $Y, C_{i}$ and $C^{\prime}$ are the fuzzy subsets of the universe $Z$, and $D_{i}$ and $D^{\prime}$ are the fuzzy subsets of the universe $W$ $(i=1,2, \ldots, N)$.

It can be seen that these rules of Table 2 are in the form of "If. . then..." statements. Each individual rule could be represented as

$$
R_{i}=A_{i} \text { AND } B_{i} \text { AND } C_{i} \rightarrow D_{i}
$$

where $R_{i}$ denotes the fuzzy relation.

According to Mamdani inference [34], we use Mamdani's product implication $A \wedge B$ for $A \rightarrow B$, where $\wedge$ denotes the minimum operator. According to (29), the fuzzy relation $\mu_{R_{i}}$ of each rule $i$ could be represented as

$$
\mu_{R_{i}}(x, y, z, w)=
$$

$$
\left[\mu_{A_{i}}(x) \wedge \mu_{B_{i}}(y) \wedge \mu_{C_{i}}(z)\right] \wedge \mu_{D_{i}}(w)
$$

where $\mu$ denotes the membership function, and $x \in X$, $y \in Y, z \in Z, w \in W$.

The overall protocol is then a relation $R$ formed by 'ORing' together with the $R_{i}$ 's:

$$
R=\bigcup_{i=1,2, \ldots, N} R_{i}
$$

Then the conclusion $D^{\prime}$ can be described as

$$
\begin{gathered}
D^{\prime}=\left(A^{\prime} \text { AND } B^{\prime} \text { AND } C^{\prime}\right) \cap R= \\
\left(A^{\prime} \operatorname{AND} B^{\prime} \operatorname{AND} C^{\prime}\right) \cap\left(\bigcup_{i=1,2, \ldots, N} R_{i}\right)= \\
D_{1}^{\prime} \cup D_{2}^{\prime} \cup \cdots \cup D_{N}^{\prime}
\end{gathered}
$$

where $\cap$ is the standard fuzzy intersection.

$$
\begin{gathered}
D_{i}^{\prime}=\left(A^{\prime} \text { AND } B^{\prime} \text { AND } C^{\prime}\right) \cap \\
{\left[\left(A_{i} \text { AND } B_{i} \text { AND } C_{i}\right) \rightarrow D_{i}\right]}
\end{gathered}
$$

And the membership function is

$$
\begin{gathered}
\mu_{D_{i}^{\prime}}(w)=\left[\mu_{A^{\prime}}(x) \wedge \mu_{A_{i}}(x)\right] \wedge \\
{\left[\mu_{B^{\prime}}(y) \wedge \mu_{B_{i}}(y)\right] \wedge} \\
{\left[\mu_{C^{\prime}}(z) \wedge \mu_{C_{i}}(z)\right] \wedge \mu_{D_{i}}(w)=\gamma_{i} \wedge \mu_{D_{i}}(w)}
\end{gathered}
$$

where $\gamma_{i}$ represents the minimum of the degrees to which the antecedents $A_{i}, B_{i}$ and $C_{i}$ are respectively compatible with the given fact $A^{\prime}, B^{\prime}$ and $C^{\prime}$.

The geometrical significance of fuzzy inference is that the conclusion $D^{\prime}$ is got by truncating each set $D_{i}$ by the value of $\gamma_{i}$ and taking the union of the truncated sets.

\subsubsection{Defuzzification}

Due to the plausibility, computational simplicity, and continuity, the center average defuzzifier is used here for defuzzification [35]. The fuzzy set $D^{\prime}$ is the union of $N$ fuzzy sets. Let $\bar{w}_{i}$ be the center of the $i$ th fuzzy set and $\mu_{\max }^{i}(w)$ be its maximal value of the membership function. The center average defuzzifier determines $w^{*}$ as

$$
w^{*}=\frac{\sum_{i=1}^{N}\left[\bar{w}_{i} \mu_{\max }^{i}(w)\right]}{\sum_{i=1}^{N} \mu_{\max }^{i}(w)} .
$$

\subsubsection{Fuzzy logic inference system}

The integrated fuzzy logic inference system has been displayed in Section 2.4.3. Through this system, we can get 
the exact value of the priority according to the exact values of the range, velocity and threat.

\subsection{Hybrid intelligent optimization algorithm}

Because the target RCS is considered as a random variable, the stochastic simulation is introduced and embedded into GA to constitute a hybrid intelligent optimization algorithm for solving the random CCP model. Hence at time $k$, we can predict the optimal time resource allocation $\boldsymbol{T}_{k+1, \text { opt }}$ of time $k+1$. The steps are as follows:

Step 1 According to the motion equation $\boldsymbol{\xi}_{k+1 \mid k}^{q}=$ $\boldsymbol{F}_{q} \cdot \boldsymbol{\xi}_{k}^{q}$ with a zero process noise, predict the state vector $\boldsymbol{\xi}_{k+1 \mid k}^{q}$ of time $k+1$.

Step 2 Calculate the priority vector $\boldsymbol{w}_{k+1}$ of all the targets by fuzzy logic inference system, and then get the complete time resource management model.

Step 3 Initialize the population, and check the feasibility of the generated chromosomes with the stochastic simulation.

Step 4 Update the chromosomes by crossover and mutation operations, and check the feasibility of the updated chromosomes with the stochastic simulation, and if they do not satisfy the constraint, correct the chromosomes.

Step 5 Calculate the objective function values of all the chromosomes, and then compute the fitness of each chromosome according to the objective function values

Step 6 Select the chromosomes by spinning the roulette wheel.

Step 7 Repeat the Steps 4-6 for a given number of cycles.

Step 8 Return the best chromosome as the optimal time allocation $\boldsymbol{T}_{k+1, \text { opt }}$.

\subsection{Target state estimation algorithm}

Based on the aforementioned solving process, in conjunction with UKF, the target state estimation algorithm for multiple target tracking is obtained. The complete procedure is described as follows:

Step 1 Let $k=1, \boldsymbol{T}_{k, \text { opt }}=\boldsymbol{T}_{0}\left(\boldsymbol{T}_{0}\right.$ denotes initial time allocation according to target detection), and for the $q$ th target, initialize $\boldsymbol{\xi}_{k-1 \mid k-1}^{q}$, the covariance $\boldsymbol{P}_{k-1 \mid k-1}^{q}=$ $\boldsymbol{J}^{-1}\left(\boldsymbol{\xi}_{k-1 \mid k-1}^{q}\right)$.

Step 2 Illuminate all the targets in line with $\boldsymbol{T}_{k, o p t}$. Then get the measurement value $\boldsymbol{z}_{k}^{q}$ of each target and calculate $\boldsymbol{R}_{\boldsymbol{\xi}_{k}^{q}}$ according to (13).

Step 3 Compute the sigma points $\chi_{i, k-1 \mid k-1}^{q}$ and their weights $\omega_{i, k}$ in accordance with the following equations:

$$
\begin{aligned}
& \left\{\begin{array}{l}
\boldsymbol{\chi}_{0, k-1 \mid k-1}^{q}=\boldsymbol{\xi}_{k-1 \mid k-1}^{q} \\
\boldsymbol{\chi}_{i, k-1 \mid k-1}^{q}=\boldsymbol{\xi}_{k-1 \mid k-1}^{q}+ \\
\quad\left(\sqrt{(I+\varsigma) \boldsymbol{P}_{k-1 \mid k-1}^{q}}\right)_{i}, \quad i=1,2, \ldots, I \\
\boldsymbol{\chi}_{i, k-1 \mid k-1}^{q}=\boldsymbol{\xi}_{k-1 \mid k-1}^{q}- \\
\quad\left(\sqrt{(I+\varsigma) \boldsymbol{P}_{k-1 \mid k-1}^{q}}\right)_{i-I}, \quad i=I+1, I+2, \ldots, 2 I
\end{array}\right. \\
& \left\{\begin{array}{l}
\omega_{0, k}=\frac{\varsigma}{I+\varsigma} \\
\omega_{i, k}=\frac{1}{2(I+\varsigma)}, \quad i=1,2, \ldots, 2 I
\end{array}\right.
\end{aligned}
$$

where $\varsigma$ is a scaling parameter, and $\left(\sqrt{(I+\varsigma) \boldsymbol{P}_{k-1 \mid k-1}^{q}}\right)_{i}$ is the $i$ th row or column of the matrix square root, $I$ is the dimension of the state vector, and $\omega_{i, k}$ is the weight that is associated with the $i$ th point.

Step 4 Transform each sigma point through the motion equation, and calculate the mean $\boldsymbol{\xi}_{k \mid k-1}^{q}$ and covariance $\boldsymbol{P}_{k \mid k-1}^{q}$ :

$$
\left\{\begin{array}{l}
\boldsymbol{\chi}_{i, k \mid k-1}^{q}=\boldsymbol{F}_{q} \cdot \boldsymbol{\chi}_{i, k-1 \mid k-1}^{q} \\
\boldsymbol{\xi}_{k \mid k-1}^{q}=\sum_{i=0}^{2 I}\left(\omega_{i} \cdot \boldsymbol{\chi}_{i, k \mid k-1}^{q}\right) \\
\boldsymbol{P}_{k \mid k-1}^{q}=\sum_{i=0}^{2 I} \omega_{i}\left(\boldsymbol{\chi}_{i, k \mid k-1}^{q}-\boldsymbol{\xi}_{k \mid k-1}^{q}\right) . \\
\quad\left(\boldsymbol{\chi}_{i, k \mid k-1}^{q}-\boldsymbol{\xi}_{k \mid k-1}^{q}\right)^{\mathrm{T}}+\boldsymbol{Q}_{k-1}^{q}
\end{array} .\right.
$$

Step 5 Transform each sigma point through the measurement equation, and compute the mean $\boldsymbol{z}_{k \mid k-1}^{q}$ and covariances $\boldsymbol{P}_{z z, k}^{q}$ and $\boldsymbol{P}_{x z, k}^{q}$ :

$$
\left\{\begin{array}{l}
\boldsymbol{z}_{i, k \mid k-1}^{q}=\boldsymbol{g}\left(\boldsymbol{\chi}_{i, k \mid k-1}^{q}\right) \\
\boldsymbol{z}_{k \mid k-1}^{q}=\sum_{i=0}^{2 I} \omega_{i} \cdot \boldsymbol{z}_{i, k \mid k-1}^{q} \\
\boldsymbol{P}_{z z, k}^{q}= \\
\sum_{i=0}^{2 I} \omega_{i}\left(\boldsymbol{z}_{i, k \mid k-1}^{q}-\boldsymbol{z}_{k \mid k-1}^{q}\right)\left(\boldsymbol{z}_{i, k \mid k-1}^{q}-\boldsymbol{z}_{k \mid k-1}^{q}\right)^{\mathrm{T}}+\boldsymbol{R}_{\boldsymbol{\xi}_{k}^{q}} \\
\boldsymbol{P}_{x z, k}^{q}=\sum_{i=0}^{2 I} \omega_{i}\left(\boldsymbol{\chi}_{i, k \mid k-1}^{q}-\boldsymbol{\xi}_{k \mid k-1}^{q}\right)\left(\boldsymbol{z}_{i, k \mid k-1}^{q}-\boldsymbol{z}_{k \mid k-1}^{q}\right)^{\mathrm{T}}
\end{array} .\right.
$$

Step 6 Calculate the gain matrix $\boldsymbol{K}_{k}^{q}$, and update the state vector $\boldsymbol{\xi}_{k \mid k}^{q}$ and covariance matrix $\boldsymbol{P}_{k \mid k}^{q}$.

$$
\left\{\begin{array}{l}
\boldsymbol{K}_{k}^{q}=\boldsymbol{P}_{x z, k}^{q} \cdot\left(\boldsymbol{P}_{z z, k}^{q}\right)^{-1} \\
\boldsymbol{\xi}_{k \mid k}^{q}=\boldsymbol{\xi}_{k \mid k-1}^{q}+\boldsymbol{K}_{k}^{q} \cdot\left(\boldsymbol{z}_{k}^{q}-\boldsymbol{z}_{k \mid k-1}^{q}\right) \\
\boldsymbol{P}_{k \mid k}^{q}=\boldsymbol{P}_{k \mid k-1}^{q}-\boldsymbol{K}_{k}^{q} \cdot \boldsymbol{P}_{z z . k}^{q} \cdot\left(\boldsymbol{K}_{k}^{q}\right)^{\mathrm{T}}
\end{array}\right.
$$

Step 7 According to the hybrid intelligent optimization algorithm, predict the optimal time resource allocation $\boldsymbol{T}_{k+1, \text { opt }}$ of time $k+1$. 
Step 8 Let $k=k+1$, and go to Step 2 .

\section{Simulation result and analysis}

In order to verify the validity of the proposed algorithm, some numerical simulation results are provided in this section. The parameters configuration is as follows.

Suppose the OAR is located at $(0,0) \mathrm{km}$, and three beams with the same transmitting power are generated simultaneously for tracking the targets. The effective bandwidth of the transmitting signal is $B_{k}^{q}=5 \mathrm{MHz}$, and the effective time duration of each coherent pulse is $T_{1}=$ $0.03 \mathrm{~ms}$. The carrier frequency of each beam is $f_{\mathrm{c}}=$ $10 \mathrm{GHz}$, and the wavelength is $0.03 \mathrm{~m}$. The sample interval of the tracking process is $T_{0}=3 \mathrm{~s}$, and we use 40 frames for this simulation.

The RCS of each target is viewed as a random variable, i.e., the RCS obeys a stochastic distribution. Without loss of generality, it is assumed that all the targets have the same PDF of RCS. We adopt the chi-square distribution of four degrees of freedom with mean 2.1517 [36] in Fig. 4.

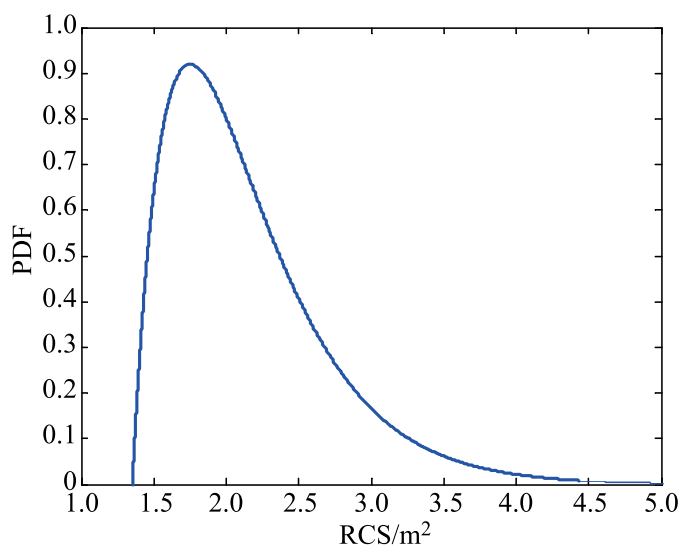

Fig. 4 PDF of $\left|h_{q}\right|$

There are $Q=9$ targets with the following parameters in Table 3.

Table 3 Parameters of all the targets

\begin{tabular}{cccc}
\hline Target & Position/km & Distance $/ \mathrm{km}$ & Velocity/(m/s) \\
\hline 1 & $(-100,60)$ & 116.62 & $(86,-51)$ \\
2 & $(-90,90)$ & 127.28 & $(354,-354)$ \\
3 & $(-80,120)$ & 144.22 & $(444,-666)$ \\
4 & $(-60,100)$ & 116.62 & $(-51,86)$ \\
5 & $(0,110)$ & 110 & $(0,-600)$ \\
6 & $(60,92)$ & 109.84 & $(164,251)$ \\
7 & $(90,104)$ & 137.54 & $(-458,-529)$ \\
8 & $(120,98)$ & 154.93 & $(-697,-569)$ \\
9 & $(120,47)$ & 128.88 & $(-186,-73)$ \\
\hline
\end{tabular}

The deployment of the targets and OAR can be seen in Fig. 5.

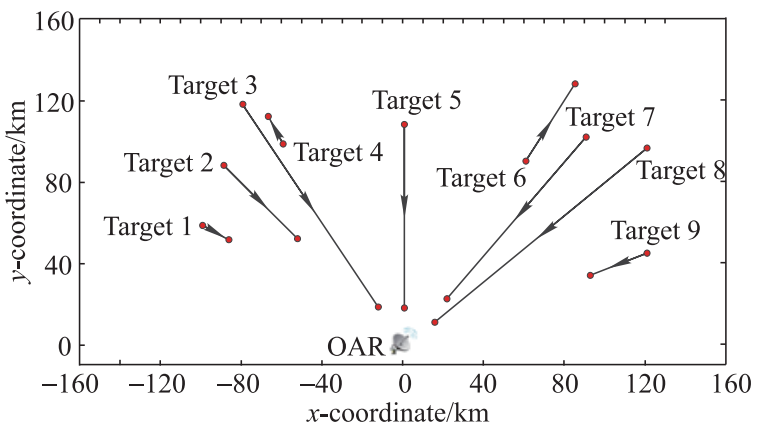

Fig. 5 Deployment of the targets and OAR

To emphasize and highlight the advantages of the resource allocation CCP, we firstly discuss and analyze the time resource CCP without the fuzzy logic priority in Situation 1. Under the condition of the same tracking error threshold, the total time for illuminating all the targets subject to different confidence levels is calculated. Then the impacts of fuzzy logic priority embedded into CCP on the time resource management of multiple target tracking are studied in Situation 2.

\subsection{Situation 1}

Due to the randomness of target RCS, the CCP can be used to balance the system resource and tracking accuracy. In other words, the probability of the RCS $\left|h_{q}\right|$ appearing around the interval endpoints is very low, so the resource programming does not need to satisfy the constraint completely, but the probability of the constraint holding is no less than a certain confidence level. As a result of this programming, large amounts of time resource will be cut down because of abandoning the extreme case that the constraint is satisfied in a low confidence level. Suppose the given total tracking error threshold is described in Fig. 6 as time goes on.

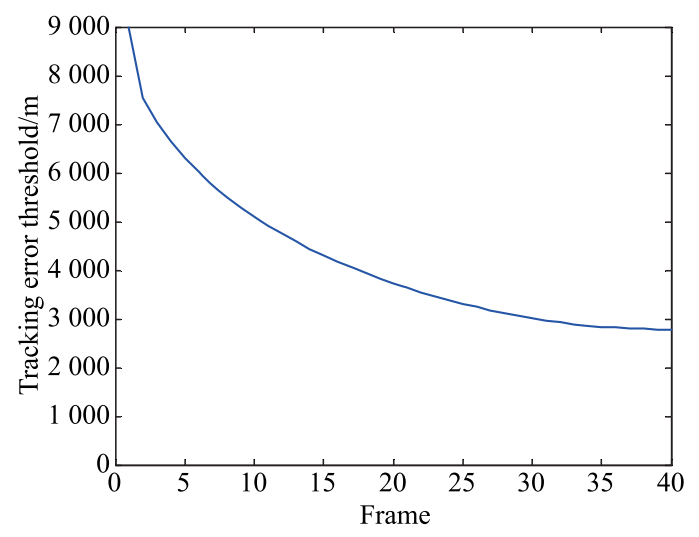

Fig. 6 Specified tracking error threshold

We set the confidence levels to $0.99,0.95$ and 0.9 , respectively. The total illuminating time of each frame is nor- 
malized by the total system time $T_{S}$.

$$
\sigma=\sum_{q=1}^{Q} T_{k}^{q} / T_{S}=\sum_{q=1}^{Q} L_{k}^{q} / L_{S}
$$

where $\sigma$ is the time resource saving rate, and $L_{S}$ is the pulse number corresponding to $T_{S}$.

On the condition that the specified confidence level is satisfied, the total illuminating time and the illuminating time of each beam can be obtained in Fig. 7 and Fig. 8, respectively.

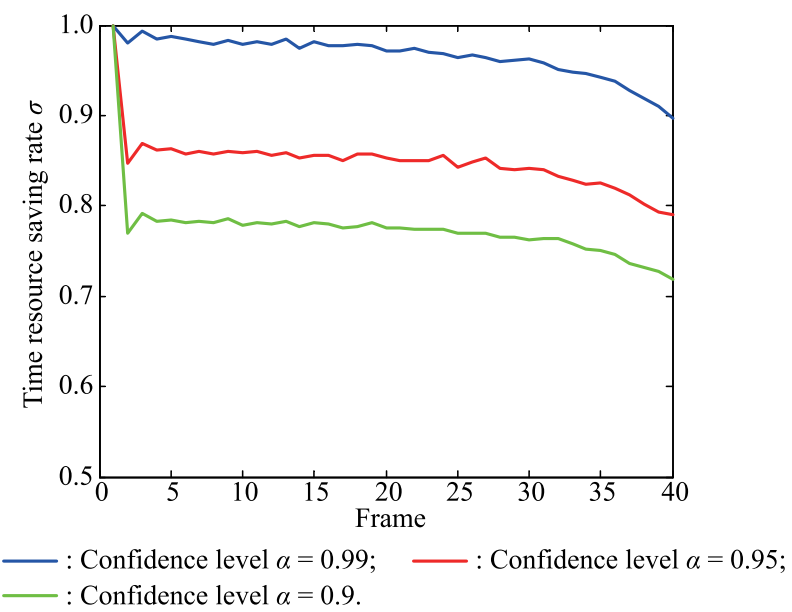

Fig. 7 Time resource saving rate of each frame

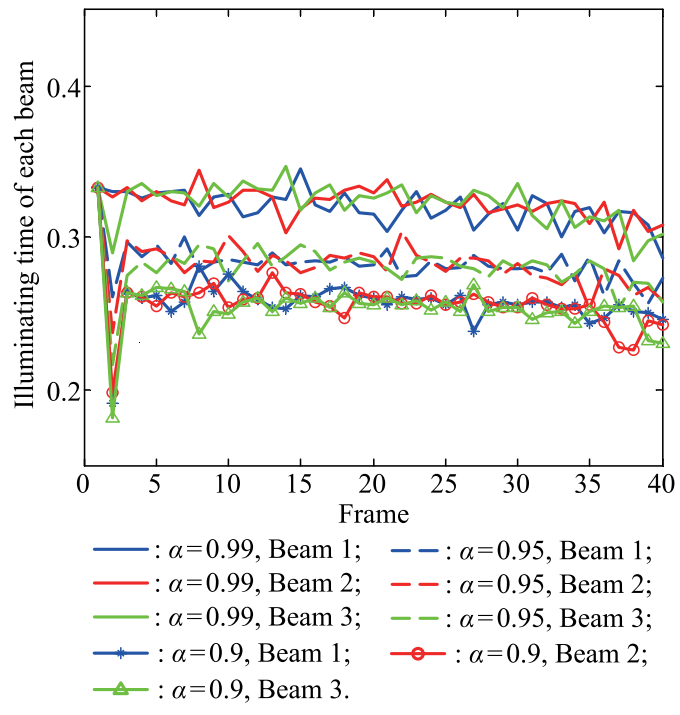

Fig. 8 Illuminating time of each beam

As can be seen in Fig. 7, the total illuminating time to all the targets is reduced following the decrease of the confidence level. When the confidence level is 0.9 , the total illuminating time is brought down by about $22 \%$. As can be also found through observation, though the tracking error threshold declines over time, the illuminating time is reducing slowly all the time instead of remaining unchanged. That is because the illuminating time for keeping the tracking error threshold decreases with the targets approaching OAR. When the tracking error threshold levels off between $35-40$ frames, the illuminating time starts to decline rapidly. The illuminating time saving rate is relative to the distribution function of target RCS.

In Fig. 8, the illuminating time of each beam under different confidence level is described. The illuminating time lessens in the wake of the decrease of the confidence level. Through the rational allocation of targets among the three beams, the illuminating time of the three beams is approximately equal.

Besides saving large time resource, the resource programming algorithm can also allocate the dwell time intelligently. The dwell time of each target is normalized by the maximum dwell time of all the targets at each frame. The dwell time allocated to each target can be shown in Fig. 9.

In Fig. 9, we can see that the target 3 and target 8 take up the most time at the beginning, and meanwhile, the target 4 and target 6 occupy the least time. The reason is that target 3 and target 8 are the farthest two, and target 4 and target 6 are the closest two at the initial time.

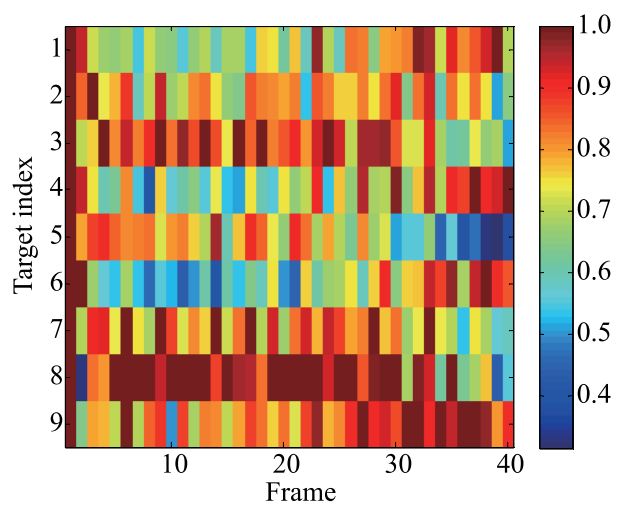

(a) $\alpha=0.99$



(b) $\alpha=0.95$ 


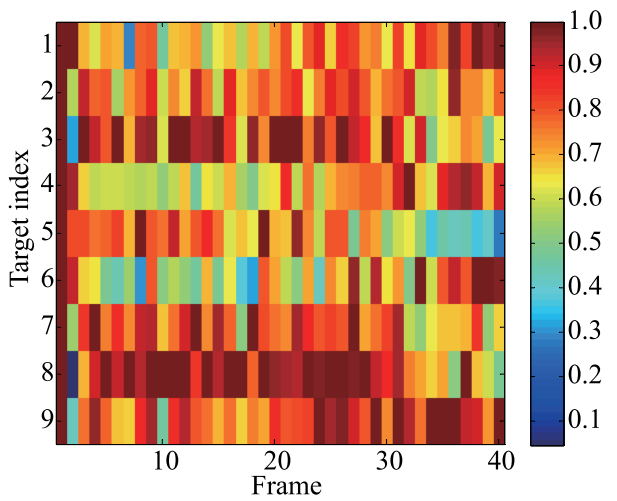

(c) $\alpha=0.9$

Fig. 9 Dwell time of each target under different confidence levels

Therefore, in order to satisfy the tracking error threshold, the dwell time allocated to target 3 and target 8 is more than the dwell time allocated to target 4 and target 6 without considering the target priority. However, as time goes by, target 3 and target 8 become the closest two, and target 4 and target 6 become the farthest two. Then the dwell time allocated to target 3 and target 8 decreases, and the dwell time allocated to target 4 and target 6 increases.

\subsection{Situation 2}

The simulation and analysis in Situation 1 ignores the priority of targets, however, as a matter of fact, the priority of targets is very important to the resource allocation of radar system. Consequently, the time resource allocation in conjunction with the priority is considered in Situation 2. The simulation parameters are the same as Situation 1. The fuzzy logic inference method is embedded into the CCP model for total illuminating time allocation. The priorities of the targets are shown in Fig. 10.

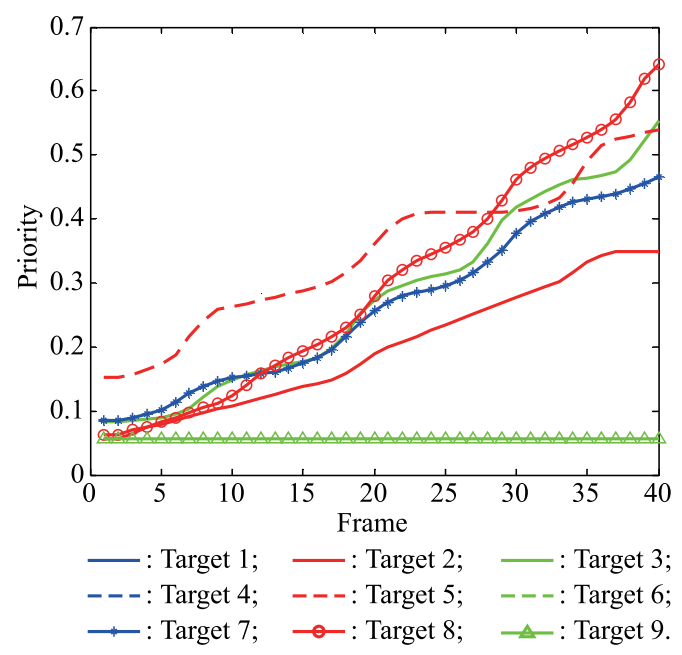

Fig. 10 Priority of the targets over time

The priority integrates the range, velocity and degree of threat. As shown in Fig. 10, the priority of each target varies continuously [20]. Hence, the changes of the targets can be reflected by priority. In regard to target 1 and target 9 , the priority is very low and changes little as time goes on for the farther distance, slower velocity and lower threat. Similar to target 1 and target 9 , the priority is very low since target 4 and target 6 are flying away from the OAR all the time. At the initial time of the tracking, target 5 has the maximum dwell time for the highest priority, however, target 3 and target 8 approach the radar with higher velocity, so the priorities of target 3 and target 8 become the highest two gradually and the radar illuminates them with the longest time.

When the confidence level is 0.9 , the total illuminating time is shown in Fig. 11 while the priority is and is not taken into account. The total illuminating time is cut down strikingly for the priority. As time goes on, the differences of the range, velocity and threat are becoming bigger and bigger. Therefore, there is no need to spend much time on the targets with low priority. The targets with high priority are always approaching the OAR. Hence, the dwell time to the targets for keeping the tracking error small is not too long.

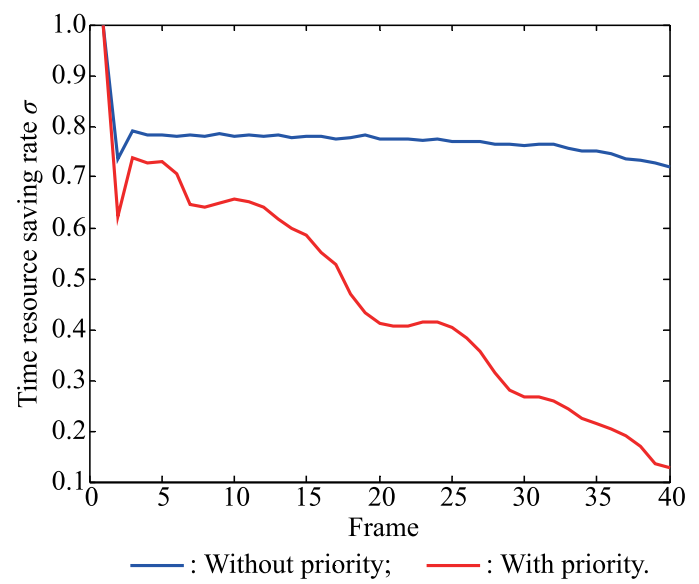

Fig. 11 Total illuminating time of all the targets

In Situation 1, all the targets have the same priority. As a consequence, the weight coefficients of the targets in (27) are all 1, i.e., $w_{k}^{q}=1$. In analogy with Situation 1, the weight coefficients in Situation 2 can be expressed in the following equation:

$$
w_{k}^{q}=Q \cdot \rho_{k}^{q} / \sum_{k=1}^{Q} \rho_{k}^{q}
$$

where $\rho_{k}^{q}$ denotes the priority. The larger the weight coefficient is, the longer the illuminating time is, so the higher the tracking accuracy is.

Fig. 12 illustrates the proportion of the illuminating time of each target. In Fig. 12(a), although the resource allocation is affected by the targets moving, compared with 
Fig. 12(b), the effect is so slight that the contrast of the dwell time on the targets is inconspicuous. However, in Fig. 12(b), when we calculate the priority of targets through fuzzy logic inference system, not only the total illuminating time is economized strikingly, but also the dwell time on each target is allocated reasonably and effectively.

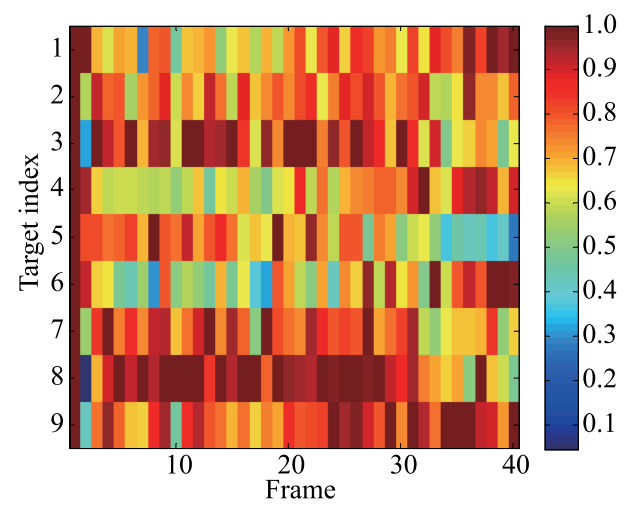

(a) Without priority

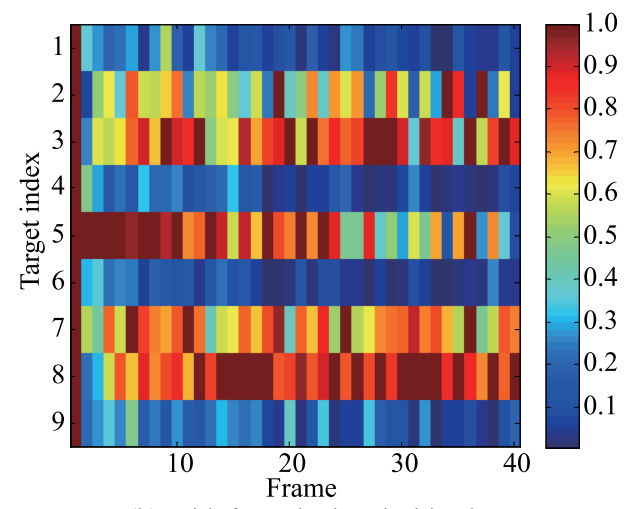

(b) With fuzzy logic prioritization

Fig. 12 Illuminating time of each target

In Fig. 13, the target indices of the longest dwell time are listed. In Fig. 13(a), when the priority is not considered, the target indices with the longest dwell time are chaotic. For instance, target 1 , target 4 , target 6 and target 9 obtain the longest dwell time at certain frames unexpectedly.

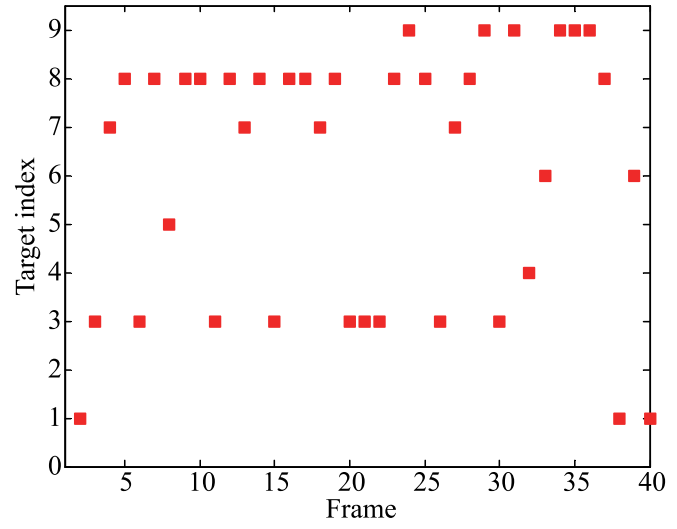

(a) Without priority

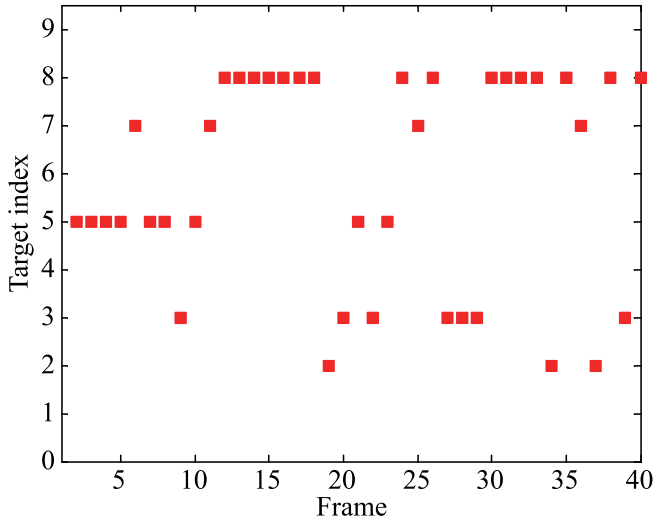

(b) With fuzzy logic prioritization

Fig. 13 Target indices with the longest illuminating time at each frame

In Fig. 13(b), the target indices with the longest dwell time concentrate upon target 2 , target 3 , target 5 , target 7 and target 8 for the high priority. More resources allocated to the closer, the faster and the more threating targets are logical.

The dwell time on the targets with low priority is short and the dwell time on the targets with high priority is long. Hence, compared with Fig. 14(a), the tracking error of the targets with high priority gets smaller in Fig. 14(b).

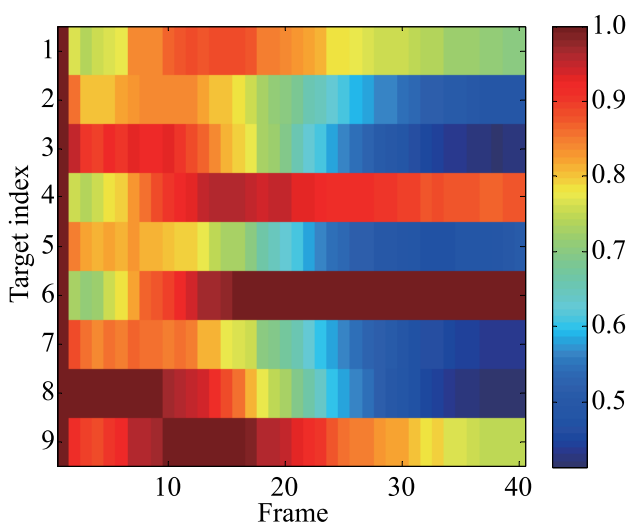

(a) Without the priority

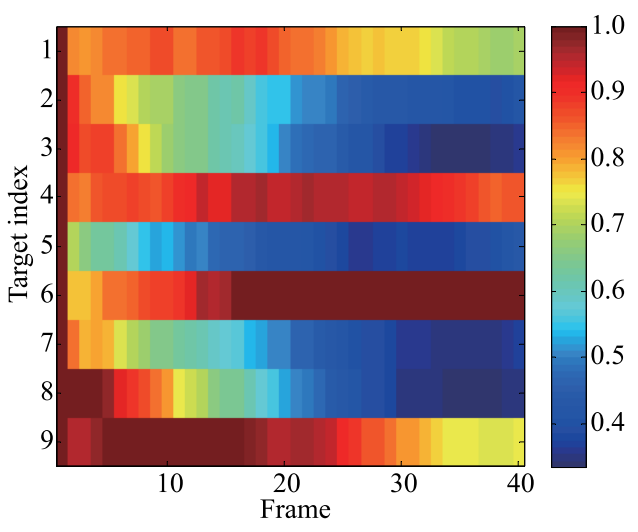

(b) With fuzzy logic priority

Fig. 14 Tracking error of each target 


\section{Conclusions and future work}

This paper proposes a time resource management scheme based on CCP in conjunction with fuzzy logic priority for multiple target tracking of OAR. The aim of this scheme is to minimize the total illuminating time conditioned on the specified confidence level. The RCS of each target is considered as a random variable, and the desired total tracking performance of the targets is deemed as the constraint. The target priority, which is computed through adaptive fuzzy logic inference system, is embedded into the hybrid intelligent optimization algorithm, which integrates stochastic simulation and GA, to calculate the optimal dwell time allocation of next frame, and then the predicted value is applied to UKF to constitute the target state estimation algorithm for solving the multiple target tracking. As seen from Situation 1, compared with the traditional deterministic resource allocation model, the CCP model can not only assure the stability of the model, but also reduce the illuminating time obviously conditioned on the confidence level. As the confidence levels decrease, the saving time resource increases. In Situation 2, considering the target priority together with $\mathrm{CCP}$, the total illuminating time can be brought down further. Moreover, the illuminating time could be allocated more reasonably over the targets. Thereby, more time resources would be saved to accomplish other tasks.

The future work will be directed to the following three aspects:

(i) The scenario of multiple target tracking in this paper is a little simple. In practical process of multiple target tracking, the target loss is a common occurrence. We need to allow for the resource management scheme when the target loss happens.

(ii) The time resource management of this paper focusing on multiple target tracking is partial. Hence, the time resource allocation of joint detection and tracking should be studied. The work of this paper lays the foundation for the time resource allocation of joint detection and tracking.

(iii) The power resource is a kind of important resource in radar system. Thus the study of joint power and the time resource management algorithm is necessary.

\section{References}

[1] BARTEE J A. Genetic algorithms as a tool for phased array radar design. Monterey, USA: Naval Postgraduate, 2002.

[2] ESSWEIN L C. Genetic algorithm design and testing of a random element 3-D 2.4 GHz phased array transmit antenna constructed of commercial RF microchips. Monterey, USA: Naval Postgraduate, 2003.

[3] KOCAMAN İ. Distributed beamforming in a swarm UAV network. Monterey, USA: Naval Postgraduate, 2008.

[4] LONG W J, BEN D, PAN M H, et al. Opportunistic digital array radar and its technical characteristic analysis. Proc. of the IET International Radar Conference, 2009: 1-4.
[5] LONG W J, BEN D, ASIM B, et al. Pattern synthesis optimization of 3-D ODAR based on improved GA using LSFE method. Journal of Harbin Institute of Technology (New Series), 2011, 18(1): 96-100.

[6] WANG Y C, WANG X, LIU H W, et al. On the design of constant modulus probing signals for MIMO radar. IEEE Trans. on Signal Processing, 2012, 60(8): 4432 - 4438.

[7] DENG H, GENG Z, HIMED B. MIMO radar waveform design for transmit beamforming and orthogonality. IEEE Trans. on Aerospace and Electronic Systems, 2016, 52(3): 1421-1433.

[8] XUE G R, DU Z C, WANG W, et al. Multi-beam dwell adaptive scheduling algorithm for helicopter-borne radar. Proc. of the 7th IEEE Joint International Information Technology and Artificial Intelligence Conference, 2014: $401-404$.

[9] SPASOJEVIC Z, DEDEO S, JENSEN R. Dwell scheduling algorithms for phased array antenna. IEEE Trans. on Asrospace and Electronic Systems, 2013, 49(1): $42-54$.

[10] GODRICH H, PETROPULU A P, POOR H V. Power allocation strategies for target localization in distributed multipleradar architectures. IEEE Trans. on Signal Processing, 2011, 59(7): $3226-3240$.

[11] GODRICH H, PETROPULU A P, POOR H V. Sensor selection in distributed multiple-radar architectures for localization: a knapsack problem formulation. IEEE Trans. on Signal Processing, 2012, 60(1): 247-260.

[12] YAN J K, LIU H W, JIN B, et al. Power allocation algorithm for target tracking in unmodulated continuous wave radar network. IEEE Sensors Journal, 2015, 15(2): $1098-1108$.

[13] SKOLNIK M. Radar handbook. 3rd ed. New York: McGrawHill Companies, 2008.

[14] CHARNES A, COOPER W W. Chance-constrained programming. Management Science, 1959: 6(1): $73-79$.

[15] BUTLER J M. Multi-function radar tracking and control. London, UK: University College London, 1998.

[16] ORMAN A J, SHAHANI A K, MOORE A R. Modelling for the control of a complex radar system. Computer \& Operations Research, 1998, 25(3): 239-249.

[17] CHENG T, HE Z S, TANG T. Dwell scheduling algorithm for multifunction phased array radars based on the scheduling gain. Journal of Systems Engineering and Electronics, 2008, 19(3): $479-485$.

[18] VINE M T. Fuzzy logic in radar resource management. Proc. of the IEE Colloquium on Multifunction Radar and Sonar Sensor Management Techniques, 2001: 1-4.

[19] MIRANDA S L C, BAKER C J, WOODBRIDGE K, et al. Knowledge-based resource management for multifunction radar. IEEE Signal Processing Magazine, 2006, 23(1): 66-76.

[20] MIRANDA S L C, BAKER C J, WOODBRIDGE K, et al. Fuzzy logic approach for prioritisation of radar tasks and sectors of surveillance in multifunction radar. IET Radar, Sonar \& Navigation, 2007, 1(2): $131-141$.

[21] TICHAVSKY P, MURAVCHIK C H, NEHORAI A. Posterior Cramér-Rao bounds for discrete-time nonlinear filtering. IEEE Trans. on Signal Processing, 1998, 46(5): 1386-1396.

[22] VAN TREES H L, BELL K L, WANG Y G. Bayesian CramérRao bounds for multistatic radar. Proc. of the IEEE International Waveform Diversity \& Design Conference, 2007: 856 859.

[23] YAN J K, JIN B, LIU H W, et al. Prior knowledge-based simultaneous multibeam power allocation algorithm for cognitive multiple targets tracking in clutter. IEEE Trans. on Signal Processing, 2015, 63(2): 512-527.

[24] YAN J K, LIU H W, JIN B, et al. Simultaneous multibeam resource allocation scheme for multiple target tracking. IEEE Trans. on Signal Processing, 2015, 63(12): 3110-3122. 
[25] YAN J K, LIU H W, JIN B, et al. Joint detection and tracking processing algorithm for target tracking in multiple radar system. IEEE Sensors Journal, 2015, 15(11): $6534-6541$.

[26] YAN J K, LIU H W, PU W Q, et al. Joint beam selection and power allocation for multiple target tracking in netted colocated MIMO radar system. IEEE Trans. on Signal Processing, 2016, 64(24): $6417-6427$.

[27] VAN TREES H L. Detection, estimation, modulation theory part III. New York: Wiley, 1971.

[28] VAN TREES H L. Optimum array processing: detection, estimation, modulation theory IV. New York: Wiley, 2001.

[29] KLIR G J, YUAN B. Fuzzy set and fuzzy logic: theory and applications. New Jersey: Prentice Hall PTR, 1995.

[30] VAN TREES H L, BELL K L. Bayesian bounds for parameter estimation and nonlinear filtering/tracking. New York: Wiley, 2007.

[31] GLASS J D, SMITH L D. MIMO radar resource allocation using posterior Cramér-Rao lower bounds. Proc. of the Aerospace Conference, 2011: 1-9.

[32] RISTIC B, ARULAMPALAM S, GORDON N. Beyond the Kalman filter: particle filters for tracking applications. Norwell, MA: Artech House, 2004.

[33] LIU B D. Theory and practice of uncertain programming. 2nd ed. Berlin: Springer-Verlag, 2009.

[34] MAMDANI E H. Application of fuzzy logic to approximate reasoning using linguistic synthesis. IEEE Trans. on Computers, 1977, 26(12): $1182-1191$.

[35] WANG L X. A course in fuzzy systems and control (International edition). New Jersey: Prentice-Hall International, 1997.

[36] SWERLING P. Radar probability of detection for some additional fluctuating target cases. IEEE Trans. on Aerospace and Electronic Systems, 1997, 33(2): 698-709.

\section{Biographies}

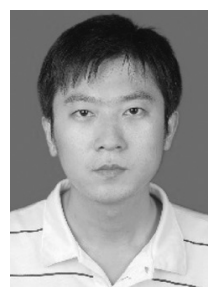

HAN Qinghua received his B.S. degree from Qingdao University of Technology in 2009 and M.S. degree from Nanjing University of Science and Technology in 2012. Now he is a Ph. D. candidate in Nanjing University of Aeronautics and Astronautics. His research interests include radar signal processing, radar resource management and new system radar.

E-mail: hanqinghua123@163.com

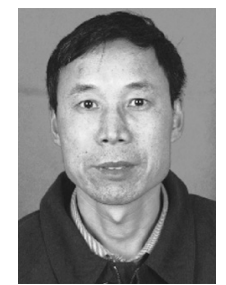

PAN Minghai received his B.S. degree in 1985 and M.S. degree in 1988 from Harbin Institute of Technology. Now he is a professor and Ph.D. supervisor of the College of Electronic and Information Engineering, Nanjing University of Aeronautics and Astronautics. His research interests include radar system design, RF simulation, radar signal processing and new system radar.

E-mail: panmh@nuaa.edu.cn

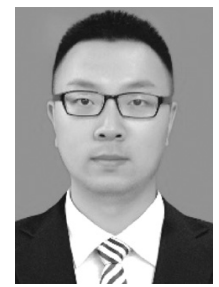

ZHANG Wucai received his B.S. degree from Nanjing University of Aeronautics and Astronautics in 2014. Now he is an M.S. candidate in Nanjing University of Aeronautics and Astronautics. His research interest is radar signal processing.

E-mail: wkyjsd@163.com

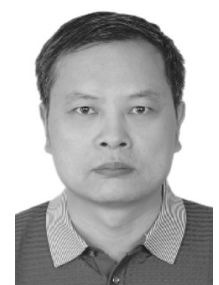

LIANG Zhiheng received his B.S. degree in 1991 and M.S. degree in 1999 from Beihang University. Now he is an associate professor of the School of Mechanical Engineering, Tsinghua University. His research interests include radio frequency simulation and radar signal processing.

E-mail: liangzhiheng@mail.tsinghua.edu.cn 